\title{
THE INTERSECTION OF SYLOW SUBGROUPS
}

\author{
AVIONAM MANN
}

ABSTRACT, Let $G$ be a finite soluble group. If the order of $G$ is not divisible by any Fermat or Mersenne primes, then there exist Sylow 2subgroups, $P$ and $Q$, such that $P \cap Q \ldots O_{p}(G)$. This improves on a result of Itô. A similar result is proved for nilpotent injectors.

Let $G$ be a finite soluble group and let $O_{p}(G)$ be the largest normal p-subgroup of $G$, In [4] Itô obtains conditions under which one can find two $p$-Sylow subgroups of $G$, say $P$ and $Q$, such that $P \cap Q=O_{p}(G)$. Some of these conditions are:

(1) $p$ is an odd non-Mersenne prime.

(2) The order $|G|$ is odd.

(3) $p=2$, and $|G|$ is not dulisible by any Fermat prime, nor by any prime $=3(\bmod 4)$.

(The other conditions in [4] relate to the structure of a Sylow $p$-subgroup of $G_{*}$ )

The object of this note is to replace (3) by the following weaker condition.

(3a) $p-2$, and $|G|$ is not divisible by any Fermat or Mersenne prime.

Proof. We imitate Itô's proof. Proceeding by induction one can, just as in [4], reduce to the case where $G=P N, P$ being a 2-Sylow subgroup of $G, N$ the unique minimal normal subgroup of $G$, and $|N|=q^{m}$ for some odd prime $q$ (in particular, $O_{2}(G)=1$ ). In this situation, to make the induc. tion step possible, we need to prove the following statement:

There exist 3 non.P.conjugate elements of $N, n_{1}, n_{2}$ and $n_{3}$, such that $P \cap n_{i}^{-1} P n_{i}=1$.

Notice that $P \cap n^{-1} P n:=C_{P}(n)$ for any $n \in N$. Still proceeding as in [4], we can assume that $P$ is nonabelian and does not contain any normal four-group. By [3, III. 7.6], $P$ has a cyclic subgroup of index 2 . Since the group $\left\langle a, b \mid a^{2^{k}}=b^{2}=1, b^{-1} a b=a^{1+2^{k-1}}\right\rangle$ contains the normal four-group $\left\langle a^{2^{k-1}}, b\right\rangle, P$ is either dihedral, generalized quaternion or quasi-dihedral. Let $|P|=2^{k}$.

Case 1. $P$ is generalized quaternion. Let $z$ be the unique involution of $P$. Since $z \notin Z(G)$ and $P$ is a maximal subgroup of $G$, we get $P=C_{G}(z)$.

Received by the editors December 19, 1974 .

AMS (MOS) subject classifications (1970). Primary 20D10, 20D20, 20D25; Secondary 20D05. 
Thus $z \notin C_{P}(n)$, for any $1 \neq n \in N$. But $z$ is contained in all the nonidentity subgroups of $P$, so $C_{P}(n)=1$. Thus, each such $n$ has $2^{k}$ conjugates. If $N$ contains only one or two classes of nonidentity elements, we obtain $q^{m}-1$ $=2^{k}$ or $q^{m}-1=2^{k+1}$, so $q^{m}$ is a Fermat prime or $q^{m}=9, q$ being a Fermat prime in either case.

Case 2. $P$ is dihedral or quasi-dihedral. Now let $z$ be the unique central involution of $P$, and let $b$ be any other involution. As above, $C_{N}(z)=1$. Also, $b$ and $b z$ are conjugate, so $\left|C_{N}(b)\right|=\left|C_{N}(b z)\right|-q^{l}$ (say). The Brauer-Wielandt formula [6] implies $m=2 l$. Thus $l$ is independent of $b$. If $c$ is an involution of $P$ different from $z$ and $b$, then $z \in\langle b, c\rangle$, so $C_{N}(b) \cap$ $C_{N}(c)=1$. If $n \in N$ and $n$ centralizes some nonidentity element in $P$, it centralizes some involution. $P$ contains exactly $2^{r}$ involutions $\neq z$, where $r=k-1$ or $k-2$. Thus, the elements of $N$ centralizing some nonidentity element of $P$ fall into $2^{r}$ subgroups of order $q^{l}$, any two such subgroups intersecting trivially. For the other elements of $N$ one has $P \cap n^{-1} P n=1$. These other elements fall into classes of order $2^{k}$, and their number is (re= call that $m=2 l$ )

$$
A=: q^{n}-1-2^{r}\left(q^{l}-1\right)-\left(q^{l} \cdots 1\right)\left(q^{l}+1-2^{r}\right) .
$$

If $A .0$, then $q^{l}=-2^{r}-1$ and $q$ is a Mersenne prime. On the other hand, if there are only one or two classes of such elements, then $\Lambda=2^{k}$ or $2^{k+1}$, in particular, $q-1 \ldots 2^{s}$ for some $s$, so $q$ is a Fermat prime.

The example at the end of [4] shows that the restriction regarding Mersenne primes is necessary. It is obvious from the proof, however, that one can allow Fermat and Mersenne primes that are big enough relative to the size of $P$. (Similarly, one can allow big Mersenne primes in 1.)

Bialostocki [1] recently proved results similar to Itô's, replacing the Sylow subgroups by nilpotent Hall subgroups. It is obvious that in these results also one can weaken the condition " $q \equiv 1(4)$ " to " $q$ is not a Mersenne prime".

Finally, we make a remark about injectors. Recall that each finite soluble group $G$ contains a unique class of subgroups, called nilpotent injectors, such that if $S$ is such an injector, and $K \triangle \triangle G$, then $S \cap K$ is a maximal nilpotent subgroup of $K([2]$ or $[5])$. Let $F(G)$ be the Fitting sub= group of $G$ For each prime $p \| G \mid$, let $C_{p}=C_{G}\left(O_{p},(F(G))\right)$, and let $S_{p}$ be any $p$-Sylow subgroup of $\left(i\right.$. Then it is known that $S=11 S_{p}$ is an injector of $G$. If we have, for each $p$, a $p$-Sylow subgroup $R_{p}$ of $C_{p}$ such that $S_{p}$ $\cap R_{p}=O_{p}(G)$, then $R \quad I_{p} R_{p}$ is also an injector and $S \cap R \ldots F(G)$. Since $C_{p} \triangle G_{\text {, }}$ we have $O_{p}\left(C_{p}\right)=C_{p} \cap O_{p}(G) \cdot O_{p}(G)$, so one can apply Itô's and our results to get:

$G$ contains two nilpotent injectors, $R$ and $S$, such that $R \cap S=F(G)$, provided at least one of the following is satisficd: 
(1) $|G|$ is odd.

(2) $|S|$ is odd ind not divisible by Mersenne primes.

(3) $|G|$ is not divisible by Fermat or Mersenne primes.

\section{REFERENCES}

1. A. Bialostocki, On products of two nilpotent subgroups of a finite group, Israel J. Math. (to appear).

2. B. Fischer, W. Gaschütz and B. Hartley, Injektoren endlicher auflösbarer Gruppen, Math.Z.102 (1967), 337-339。 MR 36 \#6504.

3. B. Huppert, Endliche Gruppen, I, Die Grundlehren der math. Wissenschaften, Band 134, Springer-Verlag, Berlin and New York, 1967. MR 37 \#302.

4. N. Itô, Uber den kleinsten p-Durchschnitt auflösbarer Gruppen, Arch. Math. 9 (1958), 27-32. MR 24 \#A1305.

5. A. Mann, Injeciors and normal subgroups of finite groups, Israel J. Math. 9 (1971), 554-558. MR $43 \# 6302$.

6. H. Wielandt, Beziehungen zwischen den Fixpunktzahlen von Automorphismengruppen einer endlichen Gruppe, Math. Z. 73 (1960), 146-158. MR 22 \#8067.

DEPARTMENT OF MATHEMATICS, HEBREW UNIVERSITY, JERUSALEM 